\title{
REFLEXIONES DESDE EL DERECHO AL MEJORAMIENTO NEURAL FARMACOLÓGICO (NEUROENHANCEMENT)* $^{*}$
}

\author{
PHARMACOLOGICAL COGNITIVE (NEUROENHANCEMENT): \\ REFLEXIONS FROM JURIDICAL PERSPECTIVE.
}

María Isabel CoRnejo PlazA*

\section{Resumen:}

En este texto se reflexiona acerca de las interfaces existentes entre una práctica novedosa denominada mejoramiento neural farmacológico en relación con las neurociencias, neuroética y neuroderecho. Describe los efectos que esta práctica produce en la cognición de sujetos sanos y, cómo su consumo está siendo problematizado por la literatura especializada, a varios niveles. La literatura destaca los aspectos éticos, de justicia distributiva y los jurídicos en las distintas fuentes del derecho, tanto a nivel funcional como conceptual.

\section{Palabras clave:}

Mejoramiento neural farmacológico, modafinil, neuroderecho, neurociencias, neuroética.

\begin{abstract}
:
This paper aims to reflect on the existing interfaces between a novel practice called pharmacological neural enhancement (neuroenhancement), concerning neuroscience, neuroethics and, neurolaw, describing the effects that this practice produces in the cognition of healthy subjects, and how its consumption is being problematized by the specialized literature, at various levels,

Artículo recibido el 5 de febrero de 2019 y aceptado para su publicación el 11 de junio de 2020.

** Doctora en Derecho, profesora de Derecho civil y Fundamentos Filosóficos del Derecho, Universidad Autónoma de Chile, correo: isabelcornejo@derechocivil.cl, ORCID: https://orcid.org/0000-0001-9116-0947.
\end{abstract}




\section{MARÍA ISABEL CORNEJO PLAZA}

among these the literature describe the ethical aspects, distributive justice aspects and the legal aspects in the different sources of law, both at a functional and conceptual level.

\section{Keywords:}

Pharmacological Cognitive Neuroenhancement, Modafinil, Neurolaw, Neurociences, Neuroethics. 
REFLEXIONES DESDE EL DERECHO AL MEJORAMIENTO NEURAL...

SumARIO: I. Introducción. II. Problema. III. Descripción de la mejora neural neuroenhancement. IV. La mejora neural farmacológica neuroenhancement en el derecho. Revisión general. V. Conclusiones. VI. Referencias.

\section{INTRODUCCIÓN}

El mejoramiento neural farmacológico es una práctica social ${ }^{1}$ que plantea importantes problemas al derecho tanto si se le concibe como sistema normativo o como parte de una disciplina que se adscribe a los problemas cognitivos de la conducta humana. En primer plano, exige adecuaciones normativas o regulatorias; en el segundo, adecuaciones conceptuales.

La investigación, tiene por objeto explorar ambos planos desde un análisis crítico efectuado desde el neuroderecho. Pretende revisar la literatura disponible esencialmente en la tradición del sistema jurídico del Common Law. ${ }^{2}$

En su conjunto, me propongo probar que esta práctica muestra desafíos y nuevos horizontes al derecho, incluso bajo un cierto supuesto, hasta un cambio de paradigma y no sólo una tensión en los

1 Mejoramiento u optimización neural farmacológica corresponde a la traducción del vocablo en inglés: neuroenhancement. En lo que sigue de este texto lo haré sinónimo de drogas inteligentes o smart drugs, utilizando indistintamente tanto la traducción castellana como los vocablos en inglés por tratarse de una práctica cuyas bases teóricas han sido estudiadas en el sistema del Common Law.

2 Existe escasa literatura en este tema emergente y específico en los sistemas jurídicos latinoamericanos. Así lo muestra un reciente estudio acerca de las últimas publicaciones referentes al neuroderecho en Latinoamérica, existiendo publicaciones solo en tres países de la región: México (17), Argentina (15) y Colombia (12). En ninguna de ellas se ha realizado investigación empírica, sino más bien discusiones relativas a la responsabilidad, libre albedrío y neuroética. La escasa base bibliográfica y la alta concentración de publicaciones provenientes de estos tres países de la región sugieren que, a pesar de su creciente impacto en países anglosajones, se necesita más trabajo en esta área para influir en la legislación de América Latina y en el desarrollo de políticas públicas. García-López, Eric et al., "Neurolaw in Latin America: Current Status and Challenges", International Journal of Forensic Mental Health, 2019, pp. 1-21. 
conceptos matrices con que trabaja el derecho: como autonomía, libre albedrío, dignidad humana, que junto a la responsabilidad conforman la agencia. El agente como señaló Aristóteles, en palabras de Ricoeur ${ }^{3}$ "es el padre de los actos, como el padre lo es de los hijos..., o bien nos remitimos al uso más primitivo de la idea de causa eficiente: ésta, expulsada de la física después de Galileo y Newton, regresa en cierto modo a su cuna, que es la experiencia del poder ejercido sobre nuestros miembros y, por intermedio de los mismos, sobre el decurso de las cosas". Locke ${ }^{4}$ insistió en que la libertad es el poder del agente, no el poder de la voluntad. Donde tal poder es, a lo menos en parte, lo que constituye el libre albedrío (free will). Los poderes de la agencia son una clase de propiedad que incluyen disposición, capacidad, habilidad, tendencia (inclinación), susceptibilidad y responsabilidad. ${ }^{5}$

El esquema general al que se ceñirá la exposición de los resultados de la investigación es el que sigue: en la introducción se ha

3 Ricoeur, Paul, Lo justo, Santiago, Chile, Jurídica, 1995, p. 29.

4 Locke, John, An Essay Concerning Human Understanding, Oxford, Oxford University Press, 1979, pp. 21 y 22.

5 Algunos poderes son lo que llamamos disposiciones causales que constan de estímulos causales, cuando estos ocurren. Como se advierte, las cuestiones relativas a la agencia presentan problemas de tipo fenomenológico, de filosofía lingüística, y hermenéutica. Ya el concepto de la libertad presenta desde la neuroética fundamental a lo menos cinco significados desde los ya famosos experimentos de Libeth publicados en 1975, lo cual puede conducirnos a distintas concepciones de libertad (principal componente de la autonomía y la agencia). Para esta investigación el impacto de los potenciadores neurales en la agencia, supone trabajar desde las concepciones del derecho tradicional de agencia, que obedecen a la concepción kantiana de autonomía, que por cierto se superponen con las concepciones epistémicas de la autonomía y agencia que están evidenciando las investigaciones de la neurociencia actual. Donde existen distintas vertientes: la determinista, emergentista, comodatista, libertaria, etcétera. El principal paradigma que derrumba la neurociencia actual es que el ser humano toma sus decisiones de acuerdo a un complejo curso causal conformado por procesos cognitivos racionales. Estas evidencias científicas por supuesto necesitan una reflexión desde el derecho, es por eso que la disciplina del neuroderecho necesita un impulso y sus propias conclusiones desde nuestra propia tradición jurídica Latinoamericana, más si consideramos que la agencia posee un fuerte componente epigenético, en que cultura y biología se unen para modelar lo que somos y podemos llegar a ser. 
descrito la hipótesis de trabajo (I); en el punto (II) se plantea el problema del mejoramiento neural farmacológico; en un (III) punto se describe la práctica del neuroenhancement y las discusiones que se han generado a la luz de la evidencia disponible en la literatura (II); posteriormente, se describe cómo esta práctica ha sido abordada por las diversas áreas del derecho (III); finalmente me referiré a las conclusiones (IV).

\section{EL PROBLEMA DEL NEUROENHANCEMENT}

El problema latente al desconocer desde el derecho la práctica del mejoramiento u optimización neural farmacológica se traduce en tensiones a varios niveles que no sólo involucran al derecho, sino cuestiones subyacentes de índole ética y política. Así, la propuesta de esta investigación consiste en un relato de las múltiples dimensiones en que el mejoramiento neural ha impactado al derecho, como la primera de muchas capas que solapan otros problemas actuales, como el individualismo. El consumo de potenciadores de la cognición es propio de la medicina del deseo en que se busca ser más inteligente, joven, bello, apelando a una subjetividad exacerbada. Si la ciencia dispone de biomejoradores morales que alteran la autonomía moral, ¿acaso ello no debería tener un correlato en lo jurídico?

La práctica del mejoramiento neural farmacológico muestra desafíos y nuevos horizontes al derecho, incluso bajo un cierto supuesto, hasta un cambio de paradigma y no solo una tensión en las concepciones morales con que trabaja el derecho: como autonomía, libre albedrío, que junto a la responsabilidad conforman la agencia. El aparato conceptual y teórico para probar esta hipótesis proviene de una metadisciplina o disciplina: el neuroderecho, ${ }^{6}$ según en qué sistema jurídico nos encontremos. Para el sistema romano germánico al cual se adscribe nuestra tradición latinoamericana, se trata más bien de una metadisciplina y en la tradición del Common Law posee más bien el carácter de disciplina propiamente tal. 171-185. 
En ese escenario, cuando hay más preguntas que respuestas el derecho se vuelca o bien a repensar sus conceptos, o bien se vale de la filosofía para pensar nuevos modelos, como es el caso de esta práctica en que no es posible homologar las soluciones jurisprudenciales de otras prácticas que se le parecen como es el caso de los desarrollos del genoma humano; o bien, otros avances científicos que encontraron soluciones jurídicas a través de los principios de respeto a la dignidad humana y al principio de protección. El problema fundamental para otorgar una respuesta al neuroenhancement es que se requiere de un modelo teórico que otorgue respuestas a quienes deseen consumir este bien suntuario, sin desconocer las diversas aristas de justicia distributiva; sin perturbar la ética del respeto a los talentos dados, protegiendo en todos los casos los derechos humanos y fundamentales de las personas (al realizarse con investigaciones éticas).

\section{DESCRIPCIÓN DE LA MEJORA NEURAL FARMACOLÓGICA NEUROENHANCEMENT}

\section{Definición de neuroenhancement}

La mejora cognitiva se ha definido como la amplificación o extensión de las capacidades centrales de la mente a través de la mejora o el aumento de los sistemas de procesamiento de información internos y externos. ${ }^{7}$ Por lo tanto, debido a que los individuos sanos están usando estos fármacos para amplificar sus capacidades cognitivas, ${ }^{8}$ ahora se los conceptualiza como una forma de mejora cognitiva y se los ha denominado potenciadores cognitivos farmacológicos (PCE). ${ }^{9}$

7 Sandberg, Ander y Savulescu, Julian, The Social and Economic Impacts of Cognitive Enhancement. In Enhancing human capacities, Oxford, Wiley-Blackwell, 2011.

8 Mohamed, Ahmed y Sahakian, Barbara, "The Ethics of Elective Psychopharmacology”, International Journal of Neuropsychopharmacology, 2011.

9 Greely, Henry, "Neuroscience and Criminal Justice: not Responsibility but Treatment”, Kansas Law Review, vol. 56, núm. 11, 2008. 
REFLEXIONES DESDE EL DERECHO AL MEJORAMIENTO NEURAL...

Se espera que los neuro-mejoradores cognitivos perfeccionen su mecanismo de acción, actualmente en el mercado se encuentra disponible el modafinil o modafinilo.

\section{Mecanismos de acción}

A continuación, se explican los efectos moleculares del modafinil, siguiendo las directrices de un reciente artículo científico que da cuenta de estudios empíricos acerca su mecanismo de acción, ${ }^{10}$ a fin de contar con la evidencia científica concreta sobre la cual basar las posteriores reflexiones. Los medicamentos que se usan para tratar los déficits cognitivos en personas con trastornos neuropsiquiátricos clínicos como la enfermedad de Alzheimer (EA), el trastorno por déficit de atención e hiperactividad (TDAH), la enfermedad de Parkinson y la esquizofrenia alteran la modulación del neurotransmisor de la cognición y mejoran la atención, el aprendizaje, la memoria y las funciones (EF) en estos pacientes. Los EF son procesos mentales que permiten a los humanos planificar, resolver problemas y pensar de forma flexible sobre cómo llevar a cabo procesos cognitivos complejos y cotidianos. La memoria de trabajo es la capacidad de mantener temporalmente activa la información relevante durante un intervalo breve y de manipularla en línea. ${ }^{11}$

Estos fármacos mejoran la cognición equilibrando los sistemas de neurotransmisores, por ejemplo, dopamina (DA) y noradrenalina (NA) de forma que restablecen algunos aspectos de la cognición en estos pacientes. Sin embargo, en entornos experimentales, la evidencia de voluntarios sanos muestra que estos fármacos mejoran las tareas cognitivas neuropsicológicas robustas en forma de U invertida y empinada, con una mejora óptima dependiendo de la dosis correcta y de tener la línea de base derecha de la función cognitiva. Las tendencias actuales muestran fuertes actitudes positivas hacia

10 Mohamed, Ahmed, "Does Modafinil Improve Cognitive Functioning in Healthy Individuals?", en Ruud ter Meulen et al. (ed.), Rethinking Cognitive Enhancement, Oxford Scholarship Online, 2017.

11 Baddeley, Alan, "Working Memory", Current Biology, 20, 2010 
el uso de estas drogas en la población general. ${ }^{12}$ Existe evidencia de que algunas personas sanas usan drogas psicoestimulantes para la mejora cognitiva ${ }^{13}$ y para mejorar el rendimiento académico. ${ }^{14}$

\section{Modafinil}

Es el nootrópico más utilizado en el mundo. Corresponde a un medicamento autorizado para la narcolepsia que tiene múltiples mecanismos de acción ${ }^{15}$ y, según informes, los adultos sanos lo utilizan como un medicamento sin prescripción médica con el objetivo de mejorar su concentración y la atención. El artículo antes mencionado revisa la evidencia de los estudios doble ciego controlados por placebo y brinda información actualizada sobre los modos de acción, los efectos cognitivos y la información de seguridad del modafinil. Evidentemente la especificidad de esta materia hace que no pueda ser incluida en este texto de manera tan exhaustiva, más allá de las propias interpretaciones y traducciones que de su lectura yo pueda llevar a las ciencias sociales, al derecho y a la neuroética, a fin de facilitar la debida comprensión de la hipótesis que pretendo demostrar.

El modafinil es un agente promotor de la vigilia autorizado para su uso en el tratamiento de la narcolepsia. ${ }^{16}$ Recientemente se aprobó para el tratamiento del "trastorno del sueño por turnos laborales". Se dice que el modafinil es una droga popular entre las personas

12 Husain, Masud y Mehta, Mitul, "Cognitive Enhancement by Drugs in Health and Disease", Trends in Cognitive Sciences, 15, 2011.

13 Farah, Martha et al., "Neurocognitive Enhancement: What Can we do and Chat Should we do? Nature Reviews", Neuroscience 5, 2004; Farah, Martha, Neuroethics: an introduction with readings, Hong Kong, MIT Press, 2010.

14 Franke, Andre Gunder et al., "Non-Medical use of Prescription Stimulants and Illicit Use of Stimulants for Cognitive Enhancement in Pupils and Students in Germany", Pharmacopsychiatry, 44, 2011.

15 Minzenberg, Michael y Carter, Cameron, "Modafinil: a Review of Neurochemical Action and Effects on Cognition", Neuropsychopharmacology, 33, 2008.

16 Benerjee, Dipavo., "Psychopharmacology for Excessive Daytime Sleepiness", Sleep Medicine Reviews, 8, 2004. 
REFLEXIONES DESDE EL DERECHO AL MEJORAMIENTO NEURAL...

sanas con el objetivo de mejorar la cognición. ${ }^{17}$ En 2004, se calculó que el $90 \%$ del modafinil se estaba utilizando sin recetas médicas en personas sanas, sin privación de sueño. ${ }^{18}$ Los ingresos globales de modafinil son más de 700 millones de dólares por año (Norman y Berger, 2008). Estas cifras no registradas e ingresos son consistentes con la afirmación de que los médicos sanos que trabajan por sistema de turnos o guardia, los pilotos de avión, los estudiantes en periodos de exámenes y académicos utilizan cada vez más modafinil para mejorar sus rendimientos cognitivos. ${ }^{19}$ Es importante mencionar que las estimaciones mencionadas anteriormente y los ingresos son extremadamente altos. Se debe tener en cuenta que, algunas de las afirmaciones hechas por los artículos de opinión no están respaldadas por las referencias citadas, y la literatura científica ${ }^{20}$ muestra evidencia mixta sobre si las personas informan usar modafinil como medicamento o como droga recreativa.

Este trabajo evidencia claramente que los estudios existentes hasta ahora no han sido efectuados con la rigurosidad requerida para realizar conclusiones fehacientes y cabales. El autor de este artículo ha señalado que en ninguno de los estudios realizados hasta ahora se ha diferenciado a los participantes por su coeficiente intelectual, lo cual podría ser problemático debido a la estigmatización que podrían sufrir aquellos con un CI más bajo del socialmente valorado. Pero, claramente los efectos del modafinil y el aderall son muy significativos en sujetos con un CI no alto y además otro factor clave es que los sujetos sometidos a investigación no han sido privados de

17 Lynch, Gary et al., "The Likelihood of Cognitive Enhancement", Pharmacology Biochemistry and Behavior, 99, 2011.

18 Baranski et al., 2004.

19 Franke, Andre Gunder et al., "Non-Medical Use of Prescription Stimulants and Illicit Use of Stimulants for Cognitive Enhancement in Pupils and Students in Germany", Pharmacopsychiatry, 44, 2011, Greely, Henry, "The Social Effects of Advances in Neuroscience: Legal Problems, Legal Prespectives. In Neuroethics: defining the issues", en Illis, J. (ed.) Theory, Practice, and Policy, Nueva York, Oxford University Press, 2006; Greely, Henry, "Neuroscience and Criminal Justice: not Responsibility but Treatment", Kansas Law Review, vol. 56, núm. 11, 2008.

20 Partridge, Bradley et al., "Smart Drugs "as Common as Coffee": Media Hype about Neuroenhancement”, PLoS ONE, 6, 2011. 
sueño. Este fármaco actúa en sujetos privados de sueño, de modo que las actuales falencias y sesgos investigativos pueden deberse entre otras cosas a la inexperiencia de los efectos del fármaco o bien a una manipulación de datos que arrojarán resultados erróneos.

No existen conclusiones fehacientes respecto de los mecanismos de acción ni las condiciones basales para lograr un óptimo efecto de estas sustancias. Se observa la existencia de variables concomitantes no adecuadamente estudiadas en estas investigaciones. Entre otras, (I) nivel de coeficiente intelectual de los participantes, (II) privación de sueño, (III) personalidad (como variable psicofisiológica, no especulación), además de uso concomitante de fármacos e interacciones farmacológicas. Es de observar que los fármacos denominados "psicofármacos" poseen efectos estrechamente dependientes del contexto de su administración, en interacción con variables psicofisiológicas del sujeto. Así, por ejemplo, el efecto de la cafeína depende de variables de personalidad y de la hora del día en que se administre. Los sujetos extravertidos suelen mejorar sus rendimientos con cafeína administrada por la mañana, en cambio los introvertidos, cuyo nivel cortical de excitación es mayor, ven decrecer sus rendimientos cuando se incrementa su nivel de excitación. Los estudios sugeridos serían fundamentales para determinar con precisión el uso no terapéutico de modafinilo y otros fármacos de esta clase, considerando todos los factores que inciden en su eficacia. Esto tiene implicaciones en cualquier generalización de sus efectos y exige un seguimiento para confirmar replicabilidad de los datos y usar las informaciones acumuladas en estudios diversos.

La investigación neuroquímica en sujetos humanos todavía no ha aportado datos empíricos suficientes para generalizar acerca de las condiciones contextuales, variables propias del sujeto, de la situación en que se administra, del efecto del grupo, de los efectos simbólicos del placebo y nocebo. Muchas sustancias utilizadas en grupos tienen efectos distintos que en situaciones de aislamiento. Lo que se relaciona en ocasiones con variables de personalidad, v.gr., los sujetos introvertidos suelen evitar los grupos demasiado interactivos porque su nivel basal de excitación cortical se supone más elevado. Esta precisión, entre otras, sugiere que los estudios clínicos y experimentales con modafinilo todavía necesitan ser refina- 
dos para dar cuenta de posibles variaciones del efecto. Si bien esto relativiza cualquier afirmación generalizadora sobre los efectos de modafinilo, ello no obsta para afirmar que en general su uso como "droga de estilo de vida" sea generalizado y los usuarios esperen mejores rendimientos (efecto placebo). No olvidemos que todo fármaco posee, además de sus efectos específicos, un efecto simbólico, atenuado o incrementado por intervenciones de otros agentes sociales (por ejemplo, el acto médico de autoridad al prescribir una intervención).

El "sistema nervioso conceptual" (esto es, la construcción teórica de valor prospectivo que emerge de estudios empíricos) propone un delicado balance entre excitación e inhibición cuyo resultado final en el comportamiento dependerá de la interacción entre las sustancias químicas, el locus o "topografía" del efecto, su duración y la interacción con otras sustancias.

Es preciso interpelar a los expertos sobre precisiones acerca de los factores que inciden en la eficacia final en estas sustancias. Esto podría incidir en una adecuada regulación y en una formulación racional de los usos sociales de ellas. Por ende, de nuestro análisis se derivarán indicaciones acerca de qué datos necesitamos para darle sentido y coherencia a los usos sociales y sugerir desarrollos necesarios para la investigación biomédica. Con el fin de impedir su mal uso como de estimular o promover un uso adecuado.

El factor edad es relevante en cualquier estudio farmacológico; el sistema nervioso humano, pese a su gran plasticidad, tiene estadios definitorios durante el desarrollo, en los cuales es más sensible a factores de estimulación e inhibición. Por ejemplo, los efectos deletéreos de los canabinoides se ha demostrado que inciden en la conectividad cerebral en forma negativa antes de los 10 años. 0 sea, las razones de prohibición de esta sustancia residen más bien en sus efectos a largo plazo (secuelas). Otro ejemplo es el alcohol, que posee un efecto acumulativo sobre el sistema nervioso lo cual se evidencia a largo plazo.

En otro plano, es indudable que los estudios deben considerar también la influencia del estado neuroendocrino en los efectos de una determinada sustancia. Para generalizar, es imperativo contar 
con estudios empíricos que consideren género, además de edad y personalidad.

\section{Neuroética de los estudios con nootrópicos}

La neuroética se inicia como disciplina a partir del congreso "Neuroethics: Mapping the Field", celebrado en San Francisco en el año 2002, organizado por las Universidades de California y Stanford con el patrocinio de la Dana Foundation. Su objetivo fue estudiar las implicaciones éticas, jurídicas y sociales, de la investigación sobre el cerebro humano. ${ }^{21} \mathrm{~W}$. Safire, conceptualiza esta nueva disciplina como "el examen de lo que es correcto o incorrecto, bueno o malo, acerca del tratamiento, perfeccionamiento, invasiones o manipulaciones del cerebro humano". ${ }^{22}$ Otra definición de neuroética señala que se refiere al "estudio de los asuntos éticos que guardan relación con el conocimiento del cerebro. De modo más preciso: la neuroética es la rama de la bioética que se preocupa de las cuestiones éticas que se suscitan a raíz de los diferentes tratamientos e intervenciones en el cerebro o en el sistema nervioso central". ${ }^{23}$ "Proviene de la intersección entre las ciencias empíricas del cerebro, la ética normativa, la filosofía de la mente, el derecho y las ciencias sociales. Se distingue entre neuroética aplicada y neuroética fundamental. La primera se aboca a los problemas prácticos, que regulan la actividad neurocientífica, constituyéndose en una rama especializada de la neurobioética. La segunda concierne al estudio (filosófico o neurocientífico) de la neurociencia de la ética. La misma se propone evaluar de qué manera el cerebro y su evolución pueden profundizar la comprensión de la identidad, autonomía, la responsabilidad, la conciencia y el yo, entre otras cuestiones". ${ }^{24}$

21 Marcus, Gary, The future of the brain, New Jersey, Princeton Press, 2015.

22 SAFIRE, William, Neuroethics. Mapping the Field, Nueva York, The Dana Press, 2002, pp. 3-9.

23 Glannon, Walter, Brain, Body and Mind. Neuroethics with a Human Face, Nueva York, Oxford University Press, 2011-14.

24 Roskies, Adina, Neuroethics for the New Millenium, National Center for Biothechnology Information, 2002, p. 21. 
Desde el punto de vista de la neuroética aplicada, al analizar la manera en que se llevan a cabo los estudios neurocientíficos, así como, la finalidad perseguida por ellos, las motivaciones de impulsar determinados estudios por sobre otros dan cuenta de una psicopolítica subyacente que persigue poner en relieve que las smart drugs son uno de los productos de la sociedad del cansancio por los altos rendimientos que nos hemos autoimpuesto. Tenemos un cambio de la dialéctica del amo y del esclavo de Hegel, en que hemos pasado a ser amos y esclavos de nosotros mismos por la propia manipulación que los medios hacen de nuestras emociones. Las emociones constituyen un medio muy eficiente de control psicopolítico de los individuos debido a que su campo de acción es dirigido directamente hacia el nivel prerreflexivo semi inconsciente de los individuos. $^{25}$

Los medios de comunicación propendiendo al consumo inherente al modelo neoliberal persuade directamente a las emociones (apelando a la libertad) logrando que sea el propio individuo quien cree que desea o necesita un producto. Además, que es él o ella quien lo elige, apelando a su valor simbólico en la cultura del rendimiento y de la eficacia.

IV. LA MEJORA NEURAL FARMACOLÓGICA O NEUROENHANCEMENT EN EL DERECHO. REVISIÓN GENERAL

\section{El Neuroderecho}

El mejoramiento neural farmacológico forma parte del estudio de una nueva disciplina denominada neuroderecho (neurolaw en el sistema del Common Law). Puede ser definida como, aquella orientación disciplinaria que se ocupa de identificar, formular y regular aquellas situaciones inéditas en que el progreso neurocientífico interpela o desafía a las instituciones sociales, las reglas y las concepciones que las inspiran. Esa interpelación posee un doble carácter:

25 Ha n, Byun-Chul, Psicopolítica: Neoliberalismo y nuevas técnicas de poder, Barcelona, Herder, 128 p. 
de una parte, es 1) funcional y, de la otra, 2) conceptual. Aunque, como se verá, de la primera puede seguirse la segunda.

1) De ellos, la literatura subraya con mayor frecuencia la relación funcional. ${ }^{26}$ En ella los hallazgos de un discurso o práctica (la neurociencia) se emplean para dilucidar problemas del otro (el derecho). ${ }^{27}$

Consideradas en forma genérica, tanto las comunidades relacionadas con el derecho como las de las neurociencias, actúan en forma separada, pero unidas pueden generar una verdadera reacción química. ${ }^{28}$ Los usos de la neurociencia para informar las cuestiones del derecho y las políticas públicas plantean el mismo tipo de cuestiones empíricas y conceptuales discutidas anteriormente con desarrollos científicos en otras áreas del conocimiento que introducen nuevos desafíos. En el ámbito empírico, los problemas son más complejos porque el derecho a menudo emplea sus propias normas para la adecuación empírica de sus reivindicaciones y, estas normas se desvían de las normas empleadas por los científicos. ${ }^{29}$

Desarrollos importantes del neuroderecho es posible hallarlos en materia penal y criminal; en la prueba pericial y testimonial; derechos fundamentales, especialmente intimidad y privacidad; en la determinación del quantum del dolor para estimar el daño moral en materia civil y la determinación de la conciencia y capacidad para discernir. En estos ámbitos la función de la neuro-tecnociencia es funcional.

2) Sin embargo, y al lado de ese servicio funcional que las neurociencias prestarían, todavía puede explorarse la relación conceptual, la manera en que uno de esos discursos modifica o tensiona las bases conceptuales del otro. Este resulta ser el caso de las tensiones que el neuroenhancement introduce en la agencia, que involucran

26 Greely, Henry, "Neuroscience and Criminal Justice: not Responsibility but Treatment", Kansas Law Review, vol. 56, núm. 11, 2008.

27 Lolas Stepke, Fernando y Cornejo Plaza, María Isabel, "Neuroética, neurociencias y derecho: culturas epistémicas y comunidades de práctica", Revista Jurisprudencia Argentina, Vol. 7, 2017, pp. 62-74.

28 Greely, Henry, "What If? The Farther Shores of Neuroethics Commentary on Neuroscience May Supersede Ethics and Law", Sci Eng Ethics, 2009.

29 Pardo, Michael y Patterson, Dennis, Minds, Brains and Law: The Conceptual Foundations of Law and Neuroscience, 2013. 
cuestiones asociadas a las concepciones de autonomía y responsabilidad.

En este caso, la investigación enmarcada dentro del neuroderecho debe servirse de la filosofía del derecho en la parte que ella describe las bases conceptuales y los supuestos fenomenológicos, hermenéuticos y metafísicos de las tradicionales concepciones jurídicas de autonomía, libertad, dignidad, identidad, etcétera, las cuales son confrontadas a la luz de los avances neurocientíficos, lo cual permite reinterpretar ideas cuya genealogía en algunos casos se remontan a la filosofía aristotélica. ${ }^{30}$

\section{Revisión del tratamiento del neuroenhancement en el derecho}

En el derecho civil de tradición romana germánica la mejora cognitiva desafía los conceptos de autonomía de la voluntad, el umbral de capacidad, las reglas de responsabilidad extracontractual, los contratos, etcétera.

En el derecho anglosajón se examinan cómo las reglas de responsabilidad (tort) pueden aplicarse a la venta y suministro de productos de mejora cognitiva, en particular dado el creciente uso no médico de éstos, incluidos los dispositivos. Existen preguntas no resueltas sobre la venta de dichos productos y la idoneidad para tal propósito, donde los medicamentos y/o dispositivos se comercializan con publicidad muchas veces exagerada sobre lo que realmente pueden lograr, que impacta en la esfera de los derechos de los consumidores. Este fenómeno, además de la necesaria discusión en la esfera de los derechos del consumidor, implica ciertamente reflexiones en el ámbito del derecho de los contratos. ${ }^{31}$

Otro punto que se ha discutido en materia civil en el área de la responsabilidad extracontractual es en lo concerniente a la negligencia, donde la mejora puede tener un impacto en el estándar del

30 Sgarbi, Adrian, "The Mystery of Freedom and Neurolaw", Beijing Law Rev, 6, 2015, pp. 133-146.

31 Ederbach, Wolfram, "Die Verbesserung des Menschen, Tatsächliche und rechtliche Aspekte der wunscherfüllenden Medizin”, MedR, núm. 26, 2008, pp. 325336. 
deber de cuidado que se espera de determinados profesionales, específicamente en ciertas especialidades médicas, como cirujanos de turno y pilotos de avión. ${ }^{32}$ Si la mejora nos permite optimizar nuestras capacidades, y si esa mejora se normaliza o generaliza (sea segura o no), esto podría tener implicaciones sobre cómo definimos el estándar de "cuidado razonable" o en nuestra tradición jurídica: "el buen padre de familia" o "persona razonable" ¿qué sucede si mejoramos nuestros tiempos de reacción? ¿O nuestra capacidad para mantener nuestra atención? Y si esto ocurre, ¿debería la ley aplicar diferentes estándares al mejorado y al no mejorado $?^{33}$ Esto nos conduce a la pregunta si sería admisible obligar a determinados profesionales a mejorarse a sí mismos, como ha sugerido la Academia de Ciencias Médicas de Londres, ${ }^{34}$ lo cual tendría claras repercusiones en la esfera de los derechos del trabajo y por supuesto en los derechos fundamentales. ${ }^{35}$

32 Grey, Betsy, "Neuroscience and Emotional Harm in Tort Law: Rethinking the American Approach to Free-Standing Emotional Distress Claim", Law and Neuroscience: Current Legal Issues, Oxford, Oxford Univ. Press., 2010.

33 Cornejo Plaza, María Isabel, "El mejoramiento neural farmacológico neuroenhancement y el impacto en la esfera de los derechos humanos ¿un desafío para la democracia?", en Valenzuela, Jonatan (ed.), Desafíos globales para la democracia, Tirant lo Blanch, pp. 39-62

34 Vincent, Nicole, "The Challenges Posed to Private Law by Emerging Cognitive Enhancement Technologies", The Law of the Future and the Future of Law, Oslo, Torkel Opsahl Academic E Publisher, 2011, p. 515.

35 Un informe reciente de las Academias Conjuntas sobre "Mejoramiento Humano y el Futuro del Trabajo", sugirió que en algunas "ocupaciones de alta responsabilidad", como la medicina y la aviación, donde las vidas de otros están en juego, "la mejora podría ser vista como una obligación moral, o incluso exigida por el público" (Academia de Ciencias Médicas et al., 2012, 38). Otro ejemplo sobre estas demandas por mejoras cognitivas es, el Mayo Journal of Clinical Proceedings (Rose y Curry, 2010:302), ambos médicos, reflexionan: “¿qué sucede si un estimulante legal que se demuestra que es seguro, se pudiese utilizar para mejorar la atención médica durante los períodos de fatiga, independientemente de la cantidad de horas trabajadas? ¿No sería la elección más ética el promover la reducción de errores...”. Con la publicación de la investigación original... el uso de medicamentos recetados para promover el estado de vigilia en ausencia de comorbilidades, como la narcolepsia y la apnea del sueño, sin duda, pasará de los militares a la arena pública. En su opinión, el uso de drogas para promover la vigilia sería el curso de acción éticamente 
REFLEXIONES DESDE EL DERECHO AL MEJORAMIENTO NEURAL...

Uno de los paradigmas emergentes en la teoría jurídica con impacto en la agencia es analizar qué tiene que decir el derecho respecto del nuevo axioma neurocientífico que afirma que no somos seres eminentemente racionales, sino más bien emocionales. ¿Cuál sería el mecanismo de acción de los potenciadores neurales en la agencia de un individuo? ¿La respuesta se debe dar sobre el modelo racional kantiano o se debe corregir hacia una epistemología con base en las neurociencias?, esta tarea forma parte de los nuevos paradigmas que la teoría jurídica con base en estudios interdisciplinarios estudia.

Otro campo innovador que toca al derecho civil en relación con el neuroenhancement en el Common Law es el problema de los potenciadores en la agencia y su real nexo causal (putativo) entre la falta de un potenciador y la contribución a un riesgo ya existente. Especial atención se ha puesto en materia de negligencia (este es el punto en materia de tort que mayor desarrollo presenta en derecho comparado) y los errores relacionados con la fatiga son la fuente más probable de posible responsabilidad civil, ya que, este es el déficit que los productos farmacéuticos han abordado con más éxito hasta la fecha. No es difícil imaginar una situación en la que un profesional, trabajando largas horas, cometa un error descuidado que probablemente esté relacionado con su fatiga. Si esa fatiga se hubiese evitado tomando una píldora, entonces es posible que el no hacerlo se considere negligente. En el Common Law, existe escasa investigación en sede jurisprudencial que ayude a determinar cómo se aplicará la prueba del "But-for", ya que, el impacto causal de la fatiga rara vez se cuestiona: cuando la fatiga es extrema se supone su efecto causal, como en los casos de los conductores que se quedan dormidos al volante. En otros casos, la atención se centra en la cuestión de la pre-

más correcto en la medicina. Warren y otros cirujanos que escriben en el Journal of Surgical Research, han apoyado tal postura y sugirieron que la idea de que los cirujanos tengan que tomar potenciadores pueden llegar a ser una práctica requerida. Ellos escriben (Warren, 2009:167-72): "La posibilidad de que los cirujanos fatigados tomen una ... droga ... para permitirles operar durante más tiempo, y posiblemente a un nivel más alto, tal vez no sea tan exagerada como algunos puedan sugerir. [Modafinil] ya se ha probado en los médicos de urgencias cuando se realizan tareas no relacionadas con la medicina al final de un turno de noche". 
visibilidad y, en si el conductor sabía o debía saber que era probable que se quedara dormido o cometiera errores debido a la fatiga. En caso afirmativo, el conductor debería haber dejado de conducir, de modo que no hacerlo corresponde a una infracción, o equivale a una conducción imprudente. En tales casos, prever que el agente está o estará fatigado equivale a entender que está exponiendo a otros a un riesgo de daño. Sin embargo, los cuestionamientos acerca de la causalidad rara vez se mencionan y no se ha encontrado una exploración detallada de la relación de causalidad-fatiga en estos casos. ${ }^{36}$ La relación causal entre la fatiga mientras se conduce y los malos resultados es aparentemente asumido a pesar de la literatura que sugiere que, al igual que con el rendimiento quirúrgico, esa relación no es tan obvia como puede pensarse. Por lo tanto, gran parte de este análisis procede de la aplicación de las reglas de causalidad con poca referencia a la discusión judicial de la causalidad y la fatiga.

La fatiga opera en un espectro, desde un pequeño grado de cansancio hasta niveles en los que un cirujano podría volverse excepcionalmente torpe o incluso dormirse. Tal fatiga extrema podría ser vista como un riesgo claro y adicional y podría llevar a una muy mala decisión del tipo que se hace cuando alguien apenas está despierto. Cuando es irrefutable que la fatiga del agente del daño es tan alta, simplemente no puede haber confusión con las causas y consecuencias de la falibilidad humana. En tales casos, sería posible decir que, salvo que el cirujano se durmiera, la operación no se habría puesto en peligro. Es probable que los tribunales consideren que la fatiga es la razón por la cual se cometió el error y que tendrán poca dificultad para ver la relación causal entre el cansancio de la operación y el resultado perjudicial.

En el ámbito del derecho penal, la discusión se ha centrado en las cuestiones del consentimiento y formación de la intención y el impacto de la mejora cognitiva en ella, así como algunas de las implicaciones de una posible mejora futura para nuestra comprensión de

36 Goold, Imogen y Maslen, Hannah, “Obliging Surgeons to Enhance Negligence Liability for Uncorrected Fatigue and Problems with Proving Causation", Medical Law Review, vol. 23, núm. 3, 2014, pp. 427-454. 
REFLEXIONES DESDE EL DERECHO AL MEJORAMIENTO NEURAL...

la capacidad. ${ }^{37}$ Ha llamado la atención el uso paralelo de los potenciadores cognitivos que podrían plantear problemas en el contexto legal, ante las situaciones de castigos ¿qué pasa si un delincuente puede evitar el castigo tomando un potenciador? Chandler explora cómo la disponibilidad de drogas podría influir en el castigo de la actividad delictiva. Los tribunales han ofrecido durante algún tiempo diversos tratamientos a los reclusos como condición de liberación: programas de tratamiento de adicciones, tratamiento antilibidinal de drogas "castración química", ${ }^{38}$ y la castración quirúrgica. ${ }^{39}$ Medidas que han demostrado ser influyentes en las sentencias. Con la disponibilidad de drogas como el acetato de ciproterona que se ha utilizado para reducir el deseo sexual en delitos de connotación sexual, la posibilidad de una "solución" médica para la delincuencia con costos potencialmente menores que la reclusión resulta ser un arreglo cada vez más pertinente.

Existen numerosas formas en que los potenciadores pueden plantear problemas en el derecho penal y criminal, según qué fármacos y dispositivos se desarrollen. Tal vez podrían usarse simplemente para llevar al acusado a la capacidad requerida para ser juzgado y entender los procedimientos. Pero, ¿qué sucedería si un potenciador mejorara la empatía? ¿0 mejorara los recuerdos? No es difícil imaginar cómo estos podrían llegar a ser muy útiles en la persecución penal de un criminal que de otro modo no se arrepentiría.

En el área de los derechos humanos se constata que, si bien sus reglas no contemplan explícitamente el fenómeno del mejoramiento neural - lo que podría inducirnos a considerar que la doctrina de los derechos humanos se muestra neutral frente a esta práctica-, existen inconsistencias o insuficiencias entre las concepciones que iluminan y permiten interpretar esas reglas, por una parte, y las prácticas que el neuroenhancement hace posibles. Como es sabido, el derecho (y el derecho de los derechos humanos no es una excep-

37 Chandler, Jennifer., "Autonomy and the Unintended Legal Consequences of Emerging Neurotherapies", Neuroethics, vol. 6, núm. 6, 2013.

38 Deacon v. Canadá, Fiscal General, 2005 FC 1489, aff'd 2006 FCA 265; Stinneford 2008.

39 Russell 1996-7 y Vanderzyl 1994-5. 
ción) se compone de reglas, principios y concepciones globales que guían las interpretaciones frente a casos difíciles.

Como sugirió Carlos $\mathrm{Nino}^{40}$ la ética y los derechos humanos descansan en tres principios globales: a) autonomía, que se refiere a que cada ser humano es capaz de trazar planes de vida y emprenderlos. Esta capacidad estaría distribuida igualmente entre todos los seres humanos. En la práctica constitucional comparada este principio funda el derecho al desarrollo de la propia personalidad y el acceso a un conjunto de bienes que permiten actualizar esa capacidad; b) dignidad, señala que cada ser humano es responsable por sus actos voluntarios y nada más que por sus actos voluntarios. Este principio funda los sistemas de responsabilidad civil con base aquiliana y permite elaborar estándares de negligencia, y c) inviolabilidad, que indica que ningún ser humano puede ser empleado como un medio para alcanzar fines socialmente benéficos.

Desde luego, la distribución igual de la autonomía podría verse alterada con un consumo desigual y masivo de esos fármacos; la dignidad por la alteración de las pautas de responsabilidad exigida por la disposición de esos fármacos; la inviolabilidad porque la distribución de esos fármacos o la generalización de esas prácticas podría conducir a que algunos seres humanos, en el ámbito laboral, por ejemplo, sean empleados (por sus menores capacidades) como medios en pos de los fines de otros.

El neuroenhancement pone en cuestión el alcance de conceptos hasta ahora cruciales en la esfera de los derechos humanos y los derechos fundamentales como son el de autonomía, libre albedrío, ${ }^{41}$ dignidad, igualdad e identidad. ${ }^{42}$ Pero, yendo más allá, esta práctica problematiza una cierta idea de sujeto que constituye una base filosófica donde se tejen el plexo de los derechos subjetivos, los derechos fundamentales, los derechos humanos y el derecho privado.

40 Nino, Carlos, La constitución de la democracia deliberativa, Barcelona, Gedisa, 1997, pp. 71-97.

41 Kolber, Adam, "Free will as a matter of law", In Philosophical Foundations of Law and Neuroscience, Oxford, Oxford University Press, 2016, pp. 15-32.

42 Earp, Brian, et al., "Adicction, identity, morality", PsyArXiv, 2018, disponible en: https://psyarxiv.com/evm84/(fecha de consulta: 18 de junio de 2020). 
REFLEXIONES DESDE EL DERECHO AL MEJORAMIENTO NEURAL...

A nivel de derechos fundamentales, este desarrollo viene a tematizar tanto la libertad en la elección del consumo de estas smart drugs, como la restricción en su consumo, atentando contra la libre autodeterminación de la personalidad, así como el impacto en el ámbito de la propia dignidad e identidad modificada del sujeto consumidor, en lo que algunos han sugerido altera la propia naturaleza humana asimilable por otros a la dignidad humana. ${ }^{43}$ En efecto, los estudios de neuroética trans y post humanistas han abordado esta temática. ${ }^{44}$

En el ámbito de los derechos humanos el mejoramiento neural farmacológico posee ya un camino recorrido, en la analogía —en lo que resulta pertinente-, con toda la reglamentación perteneciente a la vorágine que significó el descubrimiento del genoma humano y sus implicaciones en los derechos de las personas, más actualmente en las discusiones sobre la edición genética, nos trae remembranzas de las ideas de eugenesia negativa. Es decir, la prohibición de utilizar estas tecnologías para modificar genes considerados socialmente defectuosos, pero no patológicos. ${ }^{45}$

43 Bostrom, Nick., "In Defense of Posthuman Dignity", Bioethics, vol. 19, 2005, pp. 202-2149.

44 Murrow, Gail y Murrow, Richard, "A Hypothetical Neurological Association between Dehumanization and Human Rights Abuses", J Law Biosci, vol. 2, núm. 2, 2015, pp. 336-364.

45 Cfr. con Savulescu, J. and Singer, P. (2019), "An Ethical Pathway for Gene Editing" Bioethics, 33, pp. 221-222. doi:10.1111/bioe.12570, a propósito de la muy justa consternación causada en el mundo científico y bioético la noticia del investigador chino Jiankui que supuestamente utilizando la técnica CRISPR cas-9 mejoró la condición genética de unas gemelas que de otro modo corrían el riesgo de heredar el VIH de su progenitor. El reproche ético está dado porque la supuesta edición no fue realizada para corregir una enfermedad genética, sino para mejorar capacidades humanas (human enhancement), además de no realizarse de acuerdo con los protocolos elementales de toda investigación seria. Este caso constituye, sin duda un claro ejemplo de cómo la práctica científica avanza más rápido, y muchas veces en contra de regulaciones porosas y anacrónicas, que los científicos miran con malestar porque detienen sus pretensiones. El grupo de la Sociedad Americana de Bioética y de la prestigiosa revista American Journal of Bioethics han señalado, que ninguna ley ni en Estados Unidos ni en el mundo podrá prevenir que casos como este sucedan. La bioética en el mundo anglosajón está actualmente discutiendo sobre la mejora humana, y los conceptos de eugenesia se desdibujan entre los terapéuticos y los estrictamente basados en la medicina del deseo o cosmética. Ca- 
La Convención sobre Derechos Humanos y Biomedicina es el primer y único instrumento legal internacional vinculante en el campo de la biomedicina. La Convención proporciona un "marco común para la protección de los derechos humanos y la dignidad humana tanto en áreas antiguas como en lo que respecta a la aplicación de la biología y la medicina". ${ }^{46}$

La Convención actúa como documento de referencia a nivel internacional y ha tenido una influencia significativa en la legislación y las prácticas a nivel nacional, incluso en aquellos Estados Miembros del Consejo de Europa que no han firmado ni ratificado la Convención. ${ }^{47}$

De hecho, la Convención es un faro para la protección de los derechos humanos en el campo biomédico fuera del contexto europeo. México, por ejemplo, está considerando la posibilidad de adherirse a la Convención de Oviedo. Otro punto a destacar es la creciente frecuencia con que el TEDH se refiere a la Convención en sus sentencias. $^{48}$

Para las pretensiones de la industria farmacéutica de la mejora cognitiva que busca modificar la línea germinal para producir efectos perdurables, incluso hereditarios, claramente el sistema robusto

plan de la Universidad de New York señala que si bien existe inversión en ingeniería genética, no existe una marco regulatorio global que regule las implicancias éticas y jurídicas de esta práctica, donde resulta evidente que la prohibición del hijo a la carta no es posible, porque si en una legislación se prohíbe, en otras legislaciones más laxas se podrá concretar, constituyendo un asunto de interés global en que científicos, bioeticistas y políticos deberán comenzar por discutir qué se entiende por mejoramiento humano, y cuáles deberían ser sus límites éticos y jurídicos.

46 O'Sullivan Siobhán, et al., "Strasbourg", Revista de Derecho y Genoma Humano. Genética, Biotecnología y Medicina Avanzada, núm. 47, julio-diciembre de 2017, disponible en: https://rm.coe.int/oviedo-conference-rapporteur-report-e/168078295c (fecha de consulta: 18 de junio de 2020).

47 Cornejo Plaza, María Isabel, "Biobancos, Desafíos en la protección de muestras almacenadas de datos con fines de investigación biomédica. Perspectiva chilena según acuerdo Trans-Pacific Partnership (TPP)", Revista de Derecho y Genoma Humano. Genética, Biotecnología y Medicina Avanzada, núm. 47, 2017.

48 Seatzu, Francesco y Fanni, Simona, "The Experience of the European Court of Human Rights with the European Convention on Human Rights and Biomedicine", Utrecht Journal of International and European Law, vol. 31, núm. 8, 2015, pp. 5-16. 
REFLEXIONES DESDE EL DERECHO AL MEJORAMIENTO NEURAL...

de protección de los derechos humanos europeo puede constituir un verdadero desafío si quiere mantener su legislación indemne a los avances de la ciencia.

\section{La matriz ética para la regulación de los neuroenhancement}

Respecto del consumo de las smart drugs debemos realizar una distinción fundamental para dilucidar cuándo estas podrían afectar a la dignidad humana. En el caso en que se obligue a una persona a su consumo sin su consentimiento estaríamos ante una vulneración no solo de su autonomía, sino, también de su dignidad. Por ejemplo, cuando ciertos trabajadores del área de la salud son obligados a consumir modafinilo para mantener la vigilia y evitar errores en un turno nocturno. Lo mismo ocurre si en los establecimientos educacionales se les obliga a los niños a consumir drogas para evitar el Síndrome de Déficit de Atención, sin un diagnóstico ni una indicación terapéutica ni ética adecuada, es decir, constantemente sometida a revisión y considerada una medida terapéutica de última ratio. Es claro que constreñir a una persona a consumir un nootrópico, es instrumentalizarlo para obtener personas con capacidades cognitivas diversas a las dadas - una verdadera forma de eugenesia- (Cornejo, 2018: 61-65).

Por su parte, claramente ante un futuro riesgo de daño a la salud es necesario aplicar el principio de precaución. Las smart drugs han sido desarrolladas y probadas en ensayos clínicos para una población específica que posee una indicación terapéutica derivada de un diagnóstico determinado. De manera que, aún no existe suficiente evidencia científica de cómo funcionan en personas que las utilizan sin el diagnóstico para las cuales fueron indicadas. La evidencia indica que los efectos físicos negativos sobre la salud son bastante menores, pero los efectos psicológicos (adicciones) y los efectos futuros son desconocidos. Así, ante tal incertidumbre, además de la necesaria información a la comunidad — considerada una obligación de resultado por nuestros tribunales-, es necesario aplicar el principio de precaución en su distribución y comercialización. 
Por su parte, el CEDH protege el derecho de cada persona a respetar la vida privada y familiar, y esto incluye la protección contra la interferencia a la integridad corporal de un individuo.

Lo anterior sugiere que las propuestas para obligar directa o indirectamente a las personas a mejorarse a sí mismas podrían ser cuestionadas según los principios de los derechos humanos.

Una persona que se vea presionada para ingerir mejoradores neurales en el lugar de trabajo (o en otro lugar) podría argumentar que una ley que permite tal presión viola el compromiso de un país con los derechos humanos. ${ }^{49}$

Como señalan Hagger y Hagger-Johnson, el artículo 8 (1) del CEDH protege la dignidad y, por lo tanto, si los rasgos de personalidad se consideran un aspecto central de la identidad, que puede ser alterado mediante mejora cognitiva intencional o no, esto podría verse como una violación de la dignidad humana.

Una respuesta a tal desafío podría provenir de las restricciones impuestas a la protección de ese derecho. Se pueden cometer infracciones a la privacidad o a la vida familiar cuando sea necesario en una sociedad democrática en interés de la seguridad nacional, la seguridad pública o el bienestar económico del país, para la prevención del orden público y/o de la comisión de delitos, para la protección de la salud, o la moral, o para la protección de los derechos y libertades de otros. ${ }^{50}$ En los casos en que la mejora estuviese prescrita en razón de proteger a una parte o a toda la población, esta excepción podría respaldar tal obligación, pero en todo caso dependerá en gran medida de los hechos particulares, el grado de riesgo que conlleva la mejora y el impacto del fracaso para mejorar a otros. En ausencia de jurisprudencia relevante, queda por ver cómo se desarrollaría tal situación. Lo que podemos decir es que cuando se considera cómo regular los potenciadores, estos derechos básicos fundamentales deben tenerse en cuenta al evaluar qué obligaciones serán aceptables y respetuosas de los principios jurídicos vigentes.

49 Hagger, Lynn y Hagger, Gareth., "Super kids: Regulating the Use of Cognitive and Psychological Enhancement in Children", Law, Innovation and Technology, vol. 3, núm. 1, 2011, p. 261.

50 Human Rights, Act 1998, Sch 1, Art 8 
REFLEXIONES DESDE EL DERECHO AL MEJORAMIENTO NEURAL...

A un nivel de justicia distributiva, el neuroenhancement radicaliza y lleva casi a un nivel de ciencia ficción las antiguas discusiones sobre desigual distribución de los bienes, produciendo desigualdad e incrementando la segregación, desde que aquellos que puedan adquirir smart drugs se encuentran mejor situados cognitivamente frente a quienes no las consumen, produciéndose una ventaja injusta de uno sobre otros, e incluso una "selección artificial" de los mismos. Este problema de justicia social posee asimismo un dilema ético, en cuanto aquellos que están en una posición ventajosa estarían cometiendo plagio o bien dopaje intelectual, en la conocida analogía con el dopaje deportivo. En el ámbito de la ética este desarrollo ha devenido en dos vertientes claramente identificadas a saber: una corriente liberal radicalizada en el transhumanismo y el extropianismo ${ }^{51}$ que en su vertiente política se denomina posthumanismo, y que esencialmente estimula la investigación y consumo de estos bienes, exigiendo un rol decisivo al estado en la neutralización en las dificultades de acceso para todos según sus necesidades y preferencias versus una postura más bioconservadora que propone guiarse por el principio de precaución ante riesgos a la salud avizorados en investigaciones incipientes, hasta impulsar regulaciones restrictivas considerando que se afectan bienes como la integridad genética. ${ }^{52}$

A un nivel más analítico, se han hecho importantes distinciones acerca de cuándo deberían los humanos mejorarse a sí mismos. Santoni y su grupo de investigación han intentado responder esta pregunta realizando un análisis conceptual de la naturaleza de las diferentes actividades. ${ }^{53}$

Ellos señalan que la mejora cognitiva es moralmente inadmisible en algunas actividades orientadas a la práctica, como ciertas actividades educativas, v.gr. cuando la mejora cognitiva afecta negativa-

51 More, Max, Principles of extropy, Version 3.11, 2003.

52 González Morán, Luis, "Implicaciones éticas y jurídicas de las intervenciones de mejora en humanos. Reflexión general”, en Romeo Casabona, Carlos María (ed.) Más allá de la salud. Intervenciones de mejora en humanos, Bilbao, Comares, 2012, p. 14.

53 Santoni De Sio, Filippo et al., "Who Should Enhance? Conceptual and Normative Dimensions of Cognitive Enhancement", Humana. Mente Journal of Philosophical Studies, vol. 26, 2014, pp. 179-197. 
mente la esencia de esas actividades (es decir, el aprendizaje a través de un cierto tipo de esfuerzo). Por otro lado, ellos argumentan que la mejora cognitiva debe permitirse en dos grupos de casos: en primer lugar, en actividades recreativas en las que se limita el valor moral o la importancia social, en segundo término, en algunas actividades prominentemente dirigidas a objetivos, como la alta responsabilidad profesional, cuyo objetivo tiene un valor moral o social significativo. Finalmente, ellos sostienen que el uso de potenciadores cognitivos eficaces y relativamente seguros deberían incluso ser obligatorios en esas profesiones de alta responsabilidad bajo ciertas circunstancias especiales.

En el futuro, también podríamos ver la aparición de nuevos derechos humanos en respuesta a nuestras capacidades modificadas, o al menos nuestra capacidad de cambiar esas capacidades que pueden surgir con las tecnologías de mejora. ${ }^{54}$ Señalan que hasta la fecha la ley ha prestado poca atención a la protección de la interferencia con nuestros estados mentales. Argumentan que la línea entre las interferencias corporales y las interferencias mentales se atenúa en la era de las neurociencias, y que es hora de que el derecho considere cómo se pueden manejar tales interferencias. Señalan que, en la actualidad, los sistemas jurídicos distinguen entre lesiones corporales y mentales, y que esto es correcto, por lo que no deben eludirse.

En su opinión, las leyes de derechos humanos pueden proporcionar una base para protegerse contra tales interferencias. Señalan que la Unión Europea ha introducido recientemente un derecho al respeto de la "integridad mental" en el artículo 3 de la Carta de los Derechos Fundamentales. El Tribunal Europeo de Derechos Humanos incluye la integridad mental bajo el alcance del artículo 8 (privacidad), pero no ha desempeñado un papel importante (todavía). En su opinión, los problemas tales como la posibilidad de neuroenhancements no deseados (tales como la mejora de los padres a los niños contra su voluntad, o la mejora obligada en el contexto laboral), podrían abordarse a través de un derecho humano a la libre

54 Bublitz, Jan Christoph y Merkel, Reinhard, "Crimes Against Minds: On Mental Manipulations, Harms and a Human Right to Mental Self-Determination", Criminal Law and Philosophy, vol. 8, 2014, pp. 51-77. 
REFLEXIONES DESDE EL DERECHO AL MEJORAMIENTO NEURAL...

determinación (o la libertad cognitiva). Esto, sugieren, podría implementarse como una suposición subyacente dentro de la ley. Entonces, sería necesario el derecho para asegurar que las personas se encuentren protegidas de interferencias graves en su libertad mental por parte del Estado y terceros, y para mantener el derecho a su libre autodeterminación. ${ }^{55}$

Las nuevas tecnologías con promesas considerables para mejorar las capacidades humanas (y tal vez la condición humana) no pueden soslayar los riesgos concomitantes que dichos desarrollos pudieran ocasionar a futuro. Podría ser tentador ofrecer nuevas reglamentaciones específicas para gestionar estos desarrollos. Quizá en algún momento se haga necesario, pero es importante reflexionar cuidadosamente si realmente se necesitan regímenes nuevos o diferentes, o si realmente se necesita algún cambio. David Friedman ${ }^{56}$ argumenta que el capítulo tecnológico afecta al derecho al menos de tres maneras: mientras que específicamente se centren en la tecnología de mejora. Hacerlo puede llevarnos (1) a alterar el costo de violar y hacer cumplir las normas legales existentes; (2) alterar los hechos subyacentes que justifican las reglas legales, y (3) cambiar los hechos subyacentes asumidos implícitamente por la ley, volviendo obsoletos los conceptos legales y categorías existentes, o incluso sin sentido.

Las tecnologías de mejora cognitiva pueden tener los tres efectos, pero particularmente los últimos dos. El impacto potencial del uso de modafinilo en el estándar de atención por negligencia es un buen ejemplo de tales posibles impactos. Lo mismo podría aplicarse a la intencionalidad en el derecho penal, y también podríamos ver implicaciones en el área del derecho del trabajo.

Friedman destaca que las leyes ampliamente definidas rara vez, o nunca, deben adaptarse a las nuevas tecnologías, simplemente pueden abarcarlas. Pero, como continúa diciendo, estos principios a veces pueden ser demasiado amplios como para "aplicarse con resul-

55 Bublitz, Jan Christoph, "My Mind is Mine!? Cognitive Liberty as a Legal Concept”, EN Cognitive Enhancement: An Interdisciplinary Perspective, Hildt, 2013.

56 Friedman, David, “Does Technology Require New Law?", Harvard Journal of Law and Public Policy, vol. 25, núm. 2, 2001, pp. 2-71. 
tados predecibles a un costo razonable". ${ }^{57}$ Las ganancias en términos de flexibilidad y la protección del futuro debe equilibrarse con los costos de la falta de certeza y el hecho de no proporcionar a los ciudadanos una orientación clara sobre lo que es legal.

Cuando se piensa en cómo nuestras actuales legislaciones se están aplicando a la mejora cognitiva, debemos tener en cuenta los puntos de Friedman en una variedad de dimensiones. Puede ser que ya existan reglas o principios suficientemente amplios que podamos extrapolar a estos nuevos desarrollos biomédicos, por lo que podríamos aplicarlos para abordar los nuevos desafíos que nos presentan. Lo que cabe preguntarse es si las concepciones subyacentes al derecho lo permitirían. También debemos ser conscientes de no promulgar nuevas leyes (ya sea por la vía legislativa o por medio de decisiones jurisprudenciales) que operen con un alto nivel de generalidad o sancionar legislaciones que no puedan aplicarse de manera apropiada con suficiente especificidad, y que, sólo sirvan para crear o incrementar un problema jurídico e inclusive político.

Al determinar la existencia de una real necesidad de regulación, podemos preguntarnos, ¿cómo los potenciadores cognitivos han creado desafíos legales y cuál es su forma exacta? Podríamos considerar qué normas o hechos pueden haber afectado, y el grado en que dichos cambios significan en los enfoques o categorías legales existentes y si de hecho necesitan una actualización o descarte. Aquí, nuevamente, el ejemplo de negligencia es bueno. Si finalmente se mejora a todos, eso podría significar que el estándar de persona razonable ha cambiado fundamentalmente, pero, si sólo algunos son mejorados, y por elección, esto podría acarrear problemas de justicia social.

\section{CONCLUSIONES}

Este artículo ha tenido la pretensión de explicar el fenómeno del mejoramiento neural farmacológico desde el paradigma del neuroderecho. Es decir, desde un campo analítico conformado por las dis- 
quisiciones realizadas desde las neurociencias y la neuroética. Contribuyendo con una necesaria interpretación desde la neuroética aplicada acerca de cómo funciona el quehacer de la investigación neurocientífica y las prevenciones que necesariamente se deben tener al momento de interpretar críticamente sus objetivos.

La era del cerebro, como se ha denominado a la década pasada, ha incorporado en clave neuro múltiples formas de analizarnos y de analizar al mundo. Es por eso que, casi como un resultado natural de estos desarrollos se han rediseñado, surgido y reinventado junto a la neurociencia, la neurofilosofía, el neuromarketing, la neuroética, y también el neuroderecho. El neuroderecho viene a ser el interlocutor de las demás disciplinas en la interpelación de las neurociencias aplicadas a materias del comportamiento, cognición, y su impacto en las instituciones jurídicas, ya desde una certeza metodológicamente fundada en postulados neurotecnológicos y neurocientíficos actualizados al día de hoy. Esto no quiere decir que el rompecabezas de nuestra naturaleza agencial se encuentre completo, lamentablemente estamos lejos de eso, pero sin duda estamos más cerca que en ningún otro momento en la historia de la humanidad. Sin embargo, una descripción general muestra que tanto a nivel funcional el neuroderecho está posicionándose en diversas áreas del derecho, analizando cómo el mejoramiento neural da respuestas a solicitudes ciudadanas de control en la política criminal, en el análisis de la prueba de testigos y peritos en los juicios de jurados; en ayudar en la determinación del cuantum al analizar el dolor psíquico del daño moral, etcétera.

En los estudios de derecho comparado, en el sistema anglosajón específicamente, vimos que ya existen desarrollos dogmáticos para el problema del tort and negligence, donde la causalidad presenta controversias y se constituye como una gran área de investigación. Lo mismo para el caso de elevar el estándar de la culpa y de la responsabilidad para quienes hayan consumido biomejoradores. Cuestiones que son más bien de índole adscriptivas pueden evidenciar profundas tensiones con la respuesta del derecho tradicional, desde elevar el estándar de diligencia o cuidado por consumo de neuromejoradores, hasta masificarse entre algunas actividades de relevancia social, como la medicina. 
Otra cuestión de índole prescriptivo lo constituye la regulación idónea para esta práctica. El neuroenhancement se está analizando bajo determinados modelos teóricos como base para su ulterior regulación. En este sentido, bajo la premisa de la adulteración artificial de la agencia mediante un fármaco, otra respuesta del derecho es la de regular su consumo, pues no parece ser inocuo el fenómeno del mejoramiento neural farmacológico.

En efecto, existe preocupación desde distintos sectores, entre estos el académico anglosajón, para establecer una regulación que abarque las distintas posibilidades en juego. Para ello, se ofrece en esta investigación, una matriz que permite, según la naturaleza de las actividades de los agentes consumidores, una justificación moral y jurídica para prohibir su uso en niños, niñas y adolescentes. En estudiantes universitarios, es permisivo siempre que no adultere los fines específicos del espíritu de la enseñanza universitaria. Se exploró también la posibilidad de hacer obligatorio el consumo en ciertas circunstancias extremas a los médicos, como las que se han vivido a causa de la sindemia del COVID-19, para mitigar las consecuencias de la fatiga. En otras actividades de menor relevancia social, se puede permitir su consumo lúdico sin mayor reproche moral. Por supuesto una regulación como la propuesta debe fundarse en dos principios: el de precaución de riesgo a la salud, debido a que faltan estudios empíricos sobre los efectos futuros de los fármacos utilizados en personas sanas y el de justicia distributiva de Rawls, ya que la agencia se considera un bien común de la mayor importancia, no es el fármaco el que está en cuestión, sino la agencia la que, al distribuirse desigualmente, puede ahondar en las desigualdades sociales ya existentes.

Finalmente, es importante precisar que los estudios desde el neuroderecho no poseen una propuesta de arrogancia epistémica, sino que pretende constituir una mirada más, que puede llevarnos a conclusiones distintas a las exploradas en otras disciplinas, que por supuesto vale la pena tener en consideración para aquellos que se interesan por una epistemología de la agencia y sus consecuencias morales y jurídicas. 
REFLEXIONES DESDE EL DERECHO AL MEJORAMIENTO NEURAL...

\section{REFERENCIAS}

BAdDElEy, Alan, “Working memory”, Current Biology, 20, 2010.

BenerjeE, Dipavo., "Psychopharmacology for Excessive Daytime Sleepiness", Sleep Medicine Reviews, 8, 2004.

Bostrom, Nick., "In Defense of Posthuman Dignity", Bioethics, vol. 19, 2005.

Bublitz, Jan Christoph, "My mind is mine!? Cognitive liberty as a Legal Concept", Cognitive enhancement: An interdisciplinary perspective, Hildt, 2013.

Bublitz, Jan Christoph y Merkel, Reinhard, “Crimes Against Minds: On Mental Manipulations, Harms and a Human Right to Mental Self-Determination", Criminal Law and Philosophy, vol. 8, 2014.

Chul-Han, Byung, Psicopolítica. Neoliberalismo y nuevas técnicas de poder, Barcelona, Herder, 2014.

ChAndLer, Jennifer., "Autonomy and the unintended legal consequences of emerging neurotherapies", Neuroethics, vol. 6, núm. 6, 2013.

Clarke, Randolph y Reed, Thomas, "Free will and Agential Powers", en Shoemaker, D. (ed.), Agency and responsability, vol. 3, Oxford University press, 2015.

CoRnejo Plaza, María Isabel, "Biobancos, Desafíos en la protección de muestras almacenadas de datos con fines de investigación biomédica. Perspectiva chilena según acuerdo TransPacific Partnership (TPP)", Revista de Derecho y Genoma Humano. Genética, Biotecnología y Medicina Avanzada, núm. 47, Dykinson, 2017.

Cornejo PlazA, María Isabel, Capítulo 12: "Edición genética y eugenesia: nuevas perspectivas éticas y jurídicas”, en Martínez y Lima (ed.), Dilemas éticos y morales en embriones humanos: abordaje interdisciplinario en salud reproductiva, Ascune, 2018. 
CoRnejo PlazA, María Isabel, "El mejoramiento neural farmacológico neuroenhancement y el impacto en la esfera de los derechos humanos ¿un desafío para la democracia?", en VALENzuela, Jonatan (ed.) Desafíos Globales para la Democracia, Tirant lo Blanch, 2019.

DereK, Parfit, Reasons and Persons, Oxford, Oxford Clarendon Press, 1984.

EARP, Brian et al., "Adicction, Identity, Morality", PsyArXiv, 2018, disponible en: https://psyarxiv.com/evm84/(fecha de consulta: 18 de junio de 2020).

EBerbach, Wolfram, "Die Verbesserung des Menschen, Tatsächliche und rechtliche Aspekte der wunscherfüllenden Medizin", MedR, núm. 26, 2008.

FARAH, Martha et al., "Neurocognitive Enhancement: What Can we do and What Should we do? Nature Reviews", Neuroscience, 5, 2004.

FARAH, Martha, Neuroethics: an Introduction with Readings, Hong Kong, MIT Press, 2010.

FRANKE, Andre Gunder et al., "Non-Medical use of Prescription Stimulants and Illicit Use of Stimulants for Cognitive Enhancement in Pupils and Students in Germany", Pharmacopsychiatry, 44, 2011.

FRANKE, Andre Gunder et al., "Use of Illicit and Prescription Drugs for Cognitive or Mood Enhancement Among Surgeons", BMC Medicine, 11, 2013.

Friedman, David, “Does Technology Require New Law?", Harvard Journal of Law and Public Policy, vol. 25, núm. 2, 2001.

GARcíA-López, Eric et al., "Neurolaw in Latin America: Current Status and Challenges", International Journal of Forensic Mental Health, 2019.

GLannon, Walter, Brain, Body and Mind. Neuroethics with a Human Face, Nueva York, Oxford University Press, 2011.

Glannon, Walter, "Neuroscience, Free Will, and Responsibility," Journal of Ethics in Mental Health, 4, 2, 2009. 
Glannon, Walter, “Our Brains are not Us,” Bioethics, 23, 6, 2009.

GonZÁlEz MoRÁN, Luis, "Implicaciones éticas y jurídicas de las intervenciones de mejora en humanos. Reflexión general", en Romeo Casabona, Carlos María (ed.), Más allá de la salud. Intervenciones de mejora en humanos, Bilbao, Comares, 2012.

Goold, Imogen y MASLEN, Hannah, “Obliging Surgeons to Enhance Negligence Liability for Uncorrected Fatigue and Problems with Proving Causation", Medical Law Review, vol. 23, no. 3, 2014.

GREY, Betsy, "Neuroscience and Emotional Harm in Tort Law: Rethinking the American Approach toFree-Standing Emotional Distress Claim", Law and Neuroscience: Current Legal Issues, Oxford, Oxford Univ. Press., 2010.

Greely, Henry, "The Social Effects of Advances in Neuroscience: Legal Problems, Legal Prespectives. In Neuroethics: Defining the Issues", en ILlIs, J. (ed.) Theory, Practice, and Policy, Nueva York, Oxford University Press, 2006.

Greely, Henry, "Neuroscience and Criminal Justice: not Responsibility but Treatment", Kansas Law Review, vol. 56, núm. 11, 2008.

Greely, Henry et al., "Towards Responsible Use of Cognitiveenhancing Drugs by the Healthy", Nature, 456, 2008.

GreEly, Henry et al., "Law and the Revolution in Neuroscience: An Early Look at the Field", Akron Law Review, vol. 42, 2008.

Greely, Henry, "What If? The Farther Shores of Neuroethics Commentary on Neuroscience May Supersede Ethics and Law", Sci Eng Ethics, 2009.

HAgGer, Lynn y HAgGer, Gareth, "Super kids: Regulating the Use of Cognitive and Psychological Enhancement in Children", Law, Innovation and Technology, vol. 3, núm 1, 2011.

Hume, David, Tratado de la naturaleza humana, Buenos Aires, Orbishyspámerica, 1984.

Husain, Masud y Mehta, Mitul, "Cognitive Enhancement by Drugs in Health and Disease”, Trends in Cognitive Sciences, 15, 2011. 
KANT, Immanuel., Fundamentación de la metafísica de las costumbres, Madrid, Espasa-Calpe, 1994.

KolBer, Adam, "Free Will as a Matter of Law", In Philosophical Foundations of Law and Neuroscience, Oxford, Oxford University Press, 2016.

LEVy, Neil, "Is Neurolaw Conceptually Confused?", J Ethics, 18, 2, 2014.

Locke, John, An Essay Concerning Human Understanding, Oxford, Oxford University Press, 1979.

Lolas Stepke, Fernando y CoRnejo PlazA, María Isabel, "Neuroética, Neurociencias y Derecho: Culturas epistémicas y comunidades de práctica", Revista Jurisprudencia Argentina, vol. 7, 2017.

LYNCH, Gary, et al., "The Likelihood of Cognitive Enhancement", Pharmacology Biochemistry and Behavior, 99, 2011.

Marcus, Gary, The Future of the Brain, New Jersey, Princeton Press, 2015.

Minzenberg, Michael y CARTER, Cameron, "Modafinil: a Review of Neurochemical Action and Effects on Cognition", Neuropsychopharmacology, 33, 2008.

MoHAmed, Ahmed, "Does Modafinil Improve Cognitive Functioning in Healthy Individuals?", en Ruud ter Meulen et al. (ed.), Rethinking Cognitive Enhancement, Oxford Scholarship Online, 2017.

MoHAmed, Ahmed y Sahakian, Barbara, "The Ethics of Elective Psychopharmacology", International Journal of Neuropsychopharmacology, 2011.

More, Max, Principles of Extropy, Version 3.11, 2003.

Murrow, Gail y Murrow, Richard, "A hypothetical Neurological Association Between Dehumanization and Human Rights Abuses", J Law Biosci, vol. 2, núm. 2, 2015.

Nino, Carlos, La constitución de la democracia deliberativa, Barcelona, Gedisa, 1997. 
Norman, Claus y Berger, Mathias, "Neuroenhancment: Status Quo and Perspectives. European Archives of Psychiatry and Clinical", Neuroscience, 258, 2008.

0’Sullivan, Siobhán et al., "Strasbourg", Revista de Derecho y Genoma Humano. Genética, Biotecnología y Medicina Avanzada, núm. 47, julio-diciembre de 2017, disponible en: https://rm.coe. int/oviedo-conference-rapporteur-report-e/168078295c (fecha de consulta: 18 de junio de 2020).

PARDo, Michael y Patterson, Dennis, Minds, Brains, and Law. The Conceptual Foundations of Law and Neuroscience, Oxford, Oxford University Press, 2013.

PARTRIDGE, Bradley et al., "Smart Drugs "as Common as Coffee": Media Hype about Neuroenhancement”, PLOS ONE, 6, 2011.

Ricoeur, Paul, Lo justo, Santiago, Chile, Jurídica, 1995.

Rose, Steven, y Curry, Timothy, "Fatigue Countermeasures, and Performance Enhancement in Rresident Physicians", Mayo Clinic Proceedings, vol. 3, núm. 85, 2010.

SAFIRE, William, Neuroethics. Mapping the Field, Nueva York, The Dana Press, 2002.

SANDBERG, Ander y Savulescu, Julian, The Social and Economic Impacts of Cognitive Enhancement. In Enhancing Human Capacities, Oxford, Wiley-Blackwell, 2011.

Santoni De Sio, Filippo, Robichaud, Philip y Vincent, Nicole, "Who Should Enhance? Conceptual and Normative Dimensions of Cognitive Enhancement", Humana. Mente Journal of Philosophical Studies, vol. 26, 2014.

SAvulescu, Julian y Singer, Peter, "An Ethical Pathway for Gene Editing" Bioethics, 33, 2019.

SEATZu, Francesco y FAnni, Simona, "The Experience of the European Court of Human Rights with the European Convention on Human Rights and Biomedicine", Utrecht Journal of International and European Law, vol. 31, núm. 8, 2015.

SGARBI, Adrian, "The Mystery of Freedom and Neurolaw", Beijing Law Rev, 6, 2015. 
SMITH, Elizabeth y Farah, Martha, "Are Prescription Stimulants 'Smart Pills'? The Epidemiology and Cognitive Neuroscience of Does Modafinil Improve Cognitive Functioning in Healthy Individuals?", Psychological Bulletin 137, 2011.

Vincent, Nicole, "The Challenges Posed to Private Law by Emerging Cognitive Enhancement Technologies", The Law of the Future and the Future of Law, Oslo, Torkel Opsahl Academic E Publisher, 2011. 